\title{
Higher blood glucose and larger fluctuations detected postoperatively using continuous glucose monitoring: a preliminary study following total knee or hip arthroplasty
}

\author{
Yuki Maeda ${ }^{1,2^{*}}$ (D), Nobuo Nakamura ${ }^{2}$, Takashi Tsujimoto ${ }^{2}$ and Nobuhiko Sugano ${ }^{1}$
}

\begin{abstract}
s
Background: The control of diabetes mellitus (DM) should help reduce the incidence of periprosthetic joint infection (PJI). Self-monitoring of blood glucose (SMBG) concentration is usually undertaken at fixed time-points. Therefore, the extent of postoperative blood glucose fluctuation might be underestimated. To provide a more comprehensive assessment, continuous glucose monitoring (CGM) is beginning to be used. However, no previous studies have evaluated blood glucose concentrations using CGM following orthopedic surgery. Therefore, the differences between the maximum blood glucose concentrations measured using SMBG and CGM, and the mean amplitude of the glycemic fluctuation in patients with frank diabetes mellitus (DM) or pre-diabetes were evaluated. Blood glucose was measured in 20 patients who had undergone total hip or total knee arthroplasty (12 patients with DM and eight with pre-diabetes). Patients were fitted with a CGM device in the operating room, which was worn for 6 days postoperatively, and used to evaluate blood glucose concentration continuously. SMBG was performed simultaneously for the same period.

Results: The mean difference between the maximum blood glucose concentrations measured using SMBG and CGM was $25.0 \pm 20.3 \mathrm{mg} / \mathrm{dl}$ (range, -17 to $81 \mathrm{mg} / \mathrm{dl}$ ), with the concentrations measured using CGM tending to be higher than those measured using SMBG $(P=0.04)$. Blood glucose concentrations measured using CGM tended to be higher than those measured using SMBG until postoperative day 2, and to decrease gradually after postoperative day 4 . There were no significant differences in the standard deviation of the blood glucose concentrations between the two groups.
\end{abstract}

Conclusions: Blood glucose concentrations $>200 \mathrm{mg} / \mathrm{dl}$ and larger fluctuations were more frequently recorded using CGM than SMBG, especially until postoperative day 2. Thus, CGM is more useful for the identification of high blood glucose concentrations and larger fluctuations. However, this information was not provided in real time.

Keywords: Total joint arthroplasty, Continuous glucose monitoring, Glycemic variability, Periprosthetic joint infection

\section{Background}

Patient factors involved in the etiology of periprosthetic joint infection (PJI) have frequently been reported, and include a history of diabetes mellitus (DM) (Kunutsor et al., 2016). Control of DM helps reduce the incidence of PJI. To evaluate DM pre-operatively, hemoglobin $(\mathrm{Hb}) \mathrm{A} 1 \mathrm{c}$

\footnotetext{
* Correspondence: y-maeda@nn.em-net.ne.jp

${ }^{1}$ Department of Orthopaedic Medical Engineering, Osaka University Graduate

School of Medicine, 2-2 Yamadaoka Suita, Osaka 565-0871, Japan

${ }^{2}$ Center of Arthroplasty, Kyowakai Hospital, Suita, Japan
}

levels can be used, and postoperatively, blood glucose concentrations are used clinically. Previous studies have shown that the incidence of infection is higher when blood glucose concentrations are $>200 \mathrm{mg} / \mathrm{dl}$ postoperatively (Mraovic et al., 2011; Stryker et al., 2013; Tarabichi et al., 2017). Blood glucose concentrations are normally measured at set time points by self-monitoring (SMBG) using a glucometer, but this method does not enable the continuous assessment of postoperative blood glucose levels. To enable such an assessment, continuous glucose 
monitoring (CGM) is beginning to be used (Boom et al., 2014; Danne et al., 2017; Keenan et al., 2009), which more readily facilitates the identification of blood glucose variations. Indeed, blood glucose concentrations obtained using CGM sometimes show abnormal fluctuations or hypoglycemia, even when the values obtained using SMBG are normal (Danne et al., 2017).

A number of studies have been conducted to date in which blood glucose has been evaluated using CGM (Madhu et al., 2013; Rasbach et al., 2014; Zhou et al., 2017), but none have evaluated the variability in postoperative blood glucose concentrations using CGM following orthopedic surgery.

The aim of this study was to use CGM to evaluate the postoperative variability in blood glucose concentrations in patients who had undergone either total knee arthroplasty (TKA) or total hip arthroplasty (THA), and to evaluate the differences between the maximum blood glucose concentrations measured by SMBG and by CGM, and the mean amplitude of the glycemic fluctuation in patients with frank DM or pre-diabetes.

\section{Methods}

This prospective observational study was approved by our institutional review board (Kyowakai Research Ethics Board \#16-02). Written informed consent was obtained from all the participants. Three hundred patients who underwent THA or TKA in our hospital between October 2016 and September 2017 were screened. When a patient's fasting blood glucose was in the range $110-126 \mathrm{mg} / \mathrm{dl}$, and if they did not have a history of DM, an oral glucose tolerance test (OGTT) was performed. Pre-diabetes (borderline DM) was defined by a blood glucose of $140-199 \mathrm{mg} / \mathrm{dl}$, while DM was defined by a blood glucose of $>200 \mathrm{mg} / \mathrm{dl}$ during the OGTT (American Diabetes Association, 2017).

Twenty-six patients were diagnosed with DM or pre-diabetes among those who underwent THA or TKA. The blood glucose concentration of each patient with DM was controlled using rapid-acting insulin and/or long-lasting insulin before surgery, under the supervision of a diabetologist. Patients classified as pre-diabetic did not receive any treatment.

A CGM system comprising a soft sensor (Enlite sensor; Medtronic Minnesota, USA) and a small, light and water-proof recorder (iPro 2, Medtronic, Minnesota, USA) was used. Patients were fitted with the CGM device in their abdominal region, and subcutaneous glucose concentrations were recorded every $5 \mathrm{~min}$ for 6 days postoperatively. Caplin et al. (Caplin et al., 2003) have reported that the mean blood glucose concentration is $2.3 \mathrm{mg} / \mathrm{dl}$ higher than the mean subcutaneous concentration. However, subcutaneous glucose concentrations correlate closely with the equivalent blood glucose concentrations. Therefore, blood glucose concentrations were also measured regularly using SMBG to adjust the subcutaneous glucose values, and the corrected blood glucose values were subsequently evaluated.

First, the differences between the maximum blood glucose levels measured using SMBG and CGM, and the number of patients who recorded blood glucose concentrations $>200 \mathrm{mg} / \mathrm{dl}$ using both CGM and SMBG, were established. Two patients demonstrated substantial fluctuations in their postoperative blood glucose concentrations. Second, the mean amplitude of the glycemic fluctuations was evaluated using the standard deviations of the recorded values (Nishimura et al., 2008). The standard deviations of the blood glucose concentrations were also compared between the diabetic and pre-diabetic patients, and the relationships between the pre-operative $\mathrm{HbA1c}$ values and the glycemic fluctuations on each day following surgery were assessed.

All statistical analyses were performed using Bell Curve Ekuseru-Toukei (Social Survey Research Information Co., Ltd., Tokyo, Japan). Differences between the groups were evaluated using the paired $t$-test, Fisher's Exact test and relationships between variables were evaluated using multiple regression analysis. Statistical significance was accepted when $P<0.05$.

\section{Results}

Blood glucose concentrations could be measured for $>5$ days in 20 of the 26 patients ( 5 men and 15 women; mean age, 70.2 years). The mean pre-operative HbA1c was $6.7 \pm 1.1 \%$, and the mean pre-operative fasting blood glucose was $130.9 \pm 35.8 \mathrm{mg} / \mathrm{dl}$. Of these 20 patients, 12 were diabetic and eight were pre-diabetic. There was no significant difference in any parameter between the two groups (Table 1).

The mean difference between the maximum blood glucose concentrations measured using SMBG and CGM was $25.0 \pm 20.3 \mathrm{mg} / \mathrm{dl}$ (range, -17 to $81 \mathrm{mg} / \mathrm{dl}$ ), with the concentrations measured using CGM tending to be higher than those measured using SMBG (paired $t$-test; $P=0.04$ ) The maximum blood glucose concentrations measured using CGM were $>200 \mathrm{mg} / \mathrm{dl}$ in 16 patients (80\%) and those measured using SMBG were $>200 \mathrm{mg} / \mathrm{dl}$ in 11 patients (55\%).

Typical variations in blood glucose concentration are shown, in a patient with prediabetes and in another with DM, in order to clearly demonstrate the output of CGM. Patient 1 was an 81-year-old female pre-diabetic patient who underwent TKA, and whose postoperative blood glucose concentrations are shown in Fig. 1. Patient 2 was a 73-year-old female diabetic patient who underwent THA, and whose postoperative blood glucose concentrations are shown in Fig. 2. Patient 2 administered rapid-acting and long-lasting insulin before and after surgery. Figure 1 shows that for Patient 1 the fluctuations in blood glucose 
Table 1 Characteristics of the diabetic and prediabetic groups

\begin{tabular}{|c|c|c|c|c|c|c|}
\hline & Age (years) & $\operatorname{Sex}(n)$ & Body mass index $\left(\mathrm{kg} / \mathrm{m}^{2}\right)$ & Surgery (n) & Fasting blood glucose (mg/dl) & $\mathrm{HbA1c}(\%$ \\
\hline Diabetic group $(n=12)$ & $70.4 \pm 5.4$ & $\begin{array}{l}\text { Male } 4 \\
\text { Female } 8\end{array}$ & $26.6 \pm 4.2$ & $\begin{array}{l}\text { THA } 8 \\
\text { TKA } 4\end{array}$ & $141.4 \pm 40.5$ & $7.0 \pm 1.2$ \\
\hline Pre-diabetic group $(n=8)$ & $68.3 \pm 8.6$ & $\begin{array}{l}\text { Male } 1 \\
\text { Female } 7\end{array}$ & $25.4 \pm 4.1$ & $\begin{array}{l}\text { THA } 3 \\
\text { TKA } 5\end{array}$ & $125.8 \pm 16.5$ & $6.2 \pm 0.6$ \\
\hline$P$ value & 0.3 & $0.30^{*}$ & 0.5 & $0.21^{*}$ & 0.61 & 0.08 \\
\hline
\end{tabular}

Data are mean \pm SD or $n$ number. * Fisher's Exact test used. SD, standard deviation; THA, total hip arthroplasty; TKA, total knee arthroplasty; HbA1c, glycated hemoglobin

tended to be larger until postoperative day 2, after which they tended to decrease gradually, without the administration of insulin, which typical of the study cohort. The peak blood glucose concentrations measured using CGM in the postoperative period were higher than those measured using SMBG. The example graph for Patient 2 shows that the fluctuation in blood glucose tended to be large until postoperative day 2 , despite the administration of insulin.

The mean amplitudes of the glycemic fluctuation are shown in Table 2. The standard deviations of the blood glucose concentrations tended to be large until postoperative day 2 and then decreased gradually after postoperative day 4. There was no difference in the standard deviations between the diabetic and pre-diabetic groups (Table 2). Furthermore, the standard deviations over postoperative days 1-5 did not correlate with HbA1c (correlation coefficient, $r=-0.089,0.081,-0.15,0.251$, and -0.047 , respectively).

\section{Discussion}

Many studies have shown that DM is a risk factor for PJI (Kunutsor et al., 2016; Jämsen E, et al. 2012; Sato et al., 2017; Schierenbeck et al., 2017) and have identified associations between the development of postoperative infections and high HbA1c (Mraovic et al., 2011; Stryker et al., 2013; Tarabichi et al., 2017). In addition, others have identified perioperative hyperglycemia as a risk factor following total joint arthroplasty (Mraovic et al., 2011; Stryker et al., 2013). Mraovic et al. showed that the incidence of infection doubled when blood glucose concentrations before breakfast were $>200 \mathrm{mg} / \mathrm{dl}$ (Mraovic et al., 2011), and Stryker et al. reported that it was 3.75 times higher when the mean post-operative blood glucose was $>200 \mathrm{mg} / \mathrm{dl}$ (Stryker et al., 2013). Thus, it is important to measure and control blood glucose concentration in the perioperative period.

However, blood glucose concentrations tend to be measured only at fixed time points, usually three or four times a day at most. Thus, the extent of postoperative blood glucose fluctuation might frequently be underestimated, which motivated us to assess postoperative blood glucose concentrations continuously following TKA and THA. In the present study, blood glucose concentrations measured using CGM were higher than those measured using SMBG, and showed large fluctuations, especially until postoperative day 2 . The mean amplitude of the glycemic fluctuations was not significantly different

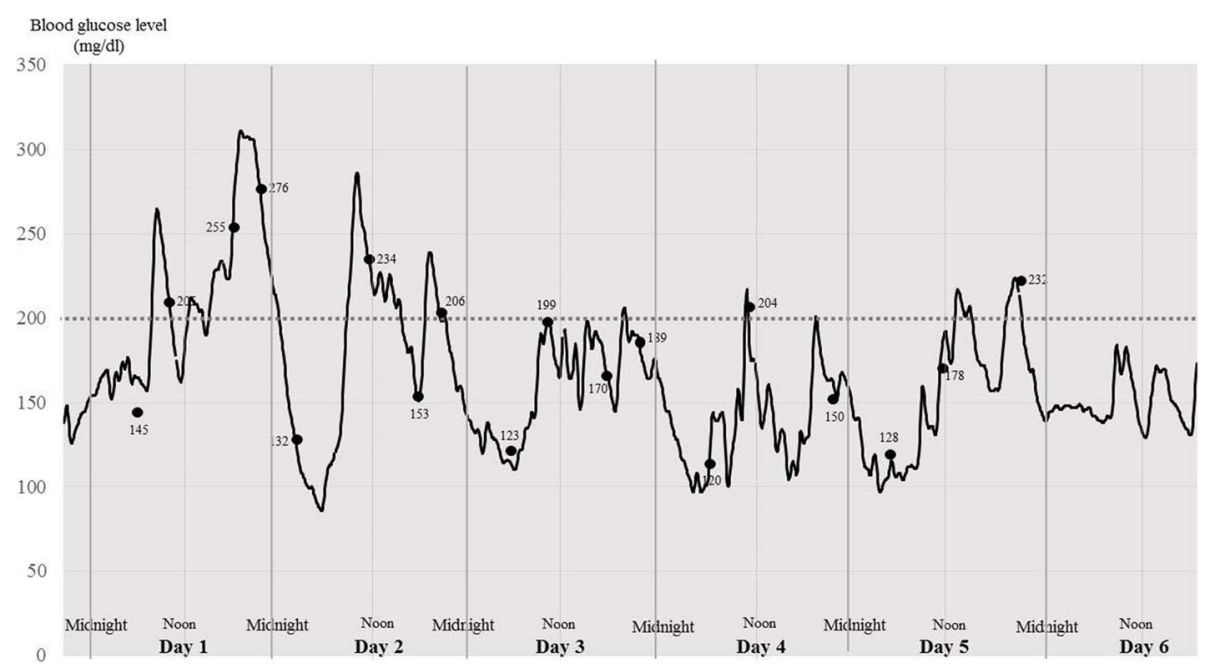

Fig. 1 Continuous blood glucose trace for Patient 1. Postoperative blood glucose concentrations in an 81-year-old woman with pre-diabetes in whom a bilateral total knee arthroplasty (TKA) had been performed. X-axis, postoperative day; Y-axis, blood glucose concentration (mg/dl). Black spots, blood glucose concentrations measured using self-monitoring of blood glucose (SMBG). Each number is the blood glucose concentration $(\mathrm{mg} / \mathrm{dl})$ at that time point 


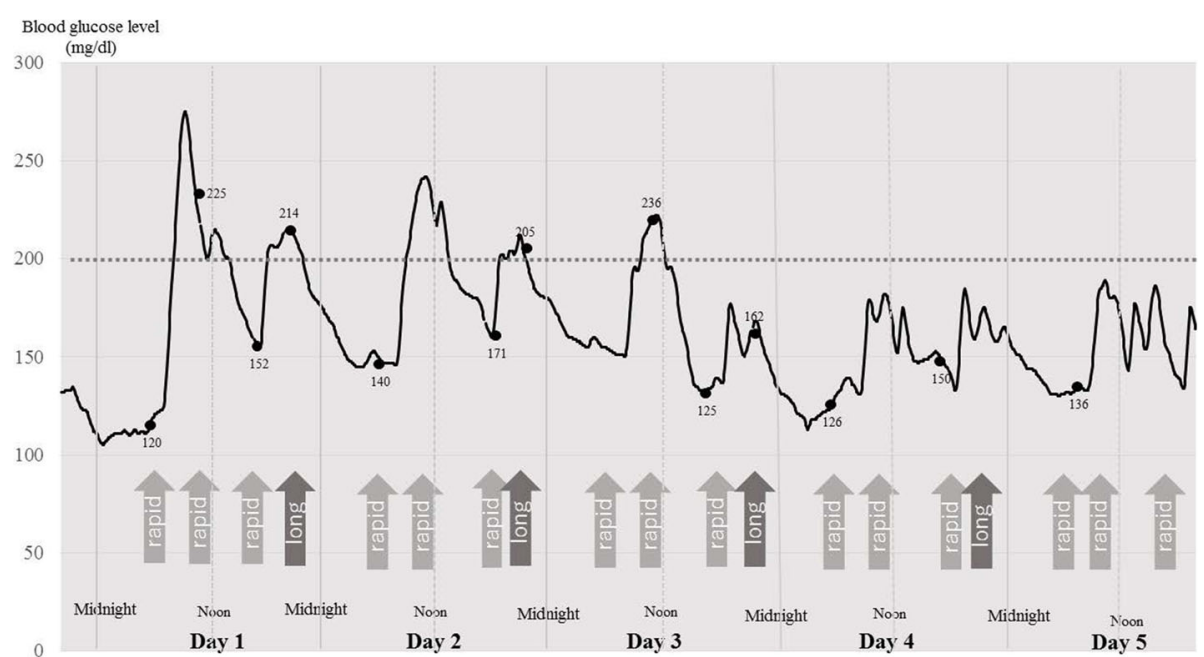

Fig. 2 Continuous blood glucose trace for Patient 2. Postoperative blood glucose in a 73-year-old woman with diabetes in whom a right total hip arthroplasty (THA) had been performed. X-axis, postoperative day; Y-axis, blood glucose concentration (mg/dl). Black spots, blood glucose concentrations measured using SMGB. Each number is the blood glucose concentration (mg/dl) at that time point. Arrow (rapid), the administration of rapid-acting insulin; arrow (long), the administration of long-acting insulin

between patients with diabetes and those with pre-diabetes, and the fluctuation in glycemia did not correlate with the pre-operative HbA1c.

According to the guidelines for the prevention of surgical site infection (SSI), a blood glucose $>200 \mathrm{mg} / \mathrm{dl}$ in the first $48 \mathrm{~h}$ following surgery is associated with a higher risk of SSI (O'Hara et al., 2018). Moreover, a blood glucose concentration $<200 \mathrm{mg} / \mathrm{dl}$ is recommended to reduce the likelihood of infection (Mraovic et al., 2011; Stryker et al., 2013). In our study, postoperative blood glucose concentrations measured using CGM reached $>200 \mathrm{mg} / \mathrm{dl}$ in $80 \%$ of the participants, but these levels were detected less frequently using SMBG. However, the blood glucose values obtained using CGM were not provided in real time. Therefore, frequent monitoring of blood glucose concentrations obtained using SMBG is currently recommended until at least postoperative day 2, not only for diabetic patients, but also for patients with pre-diabetes.

The mean amplitude of the glycemic fluctuations was not significantly different between the pre-diabetic and diabetic groups. Large fluctuations in blood glucose may be an important predictor of mortality, as well as of

Table 2 Mean amplitude of the fluctuation in glucose concentration in the diabetic and pre-diabetic groups

\begin{tabular}{llllll}
\hline & Day 1 & Day 2 & Day 3 & Day 4 & Day 5 \\
\hline All cases $(n=20)$ & 32.7 & 26.6 & 21.6 & 21.5 & 17.4 \\
Diabetic group $(n=12)$ & 35.8 & 27.7 & 17.1 & 21.5 & 15.9 \\
Pre-diabetic group $(n=8)$ & 28.8 & 25.1 & 27.3 & 21.4 & 19.3 \\
$P$ value & 0.35 & 0.65 & 0.07 & 0.99 & 0.23 \\
\hline
\end{tabular}

Data are the standard deviation of the subcutaneous glucose concentration on each post-operative day $(\mathrm{mg} / \mathrm{dl})$ major adverse cardiac events (Su et al., 2013). Although no previous publications have suggested that patients with pre-diabetes may be at greater risk of postoperative complications, including infections, our findings imply that pre-diabetic patients should be as vigilant regarding postoperative complications as diabetic patients.

We hypothesized that HbA1c level can be used as a marker of glycemic control, and is routinely measured in many patients with diabetes (Rohlfing et al., 2002). Hwang demonstrated a strong correlation between preoperative $\mathrm{HbA1c}$ and postoperative glucose concentrations in patients who underwent TKA (Hwang et al., 2015). However, in our study, the degree of postoperative blood glucose fluctuation did not correlate with HbA1c. Consistent with this, Suwa et al. reported that the $\mathrm{HbA1c}$ value did not reflect glucose fluctuation as well as the short-term mean glucose value in 43 diabetic patients (Suwa et al., 2010). Thus, other, yet to be identified, glycemic markers may prove to be more useful than HbA1c for the evaluation of fluctuations in blood glucose (Suwa et al., 2010; Shohat et al., 2017).

There were several limitations to this study. First, multivariate analysis was not performed because of the small number of participants, and for the same reason the statistical power was low for the comparison of the mean amplitude of the glycemic fluctuations between the pre-diabetic and diabetic groups. Thus, further evaluation using a larger number of patients is required. Second, blood glucose data obtained using the CGM system in this study could not provide a real-time output, such that hypoglycemia could not be rapidly detected, and blood glucose could not be measured after the removal of the CGM device. Finally, we did not evaluate 
the blood glucose concentrations of patients who developed infections or other complications following TJA. However, CGM was found to be easy to use and a useful tool for the continuous measurement of blood glucose concentrations and their fluctuation, which could not be achieved using SMBG. If the blood glucose fluctuations in patients who subsequently developed infections could be assessed, and sufficient data for such an analysis obtained, this would be helpful to define the ideal blood glucose range to minimize the risk of PJI.

\section{Conclusions}

We conclude that higher blood glucose concentrations and larger fluctuations are detected using CGM than SMBG, and that higher blood glucose concentrations exist postoperatively than were previously thought, especially during days 1-2. Thus, CGM may represent a useful tool for the prevention of extreme blood glucose concentrations, which should help to reduce the risk of PJI.

\section{Acknowledgements}

We would like to thank Ms. Yuko Uesugi, Department of International Health, Graduate School of Health Science, Kobe University for help with the study concept.

\section{Funding}

No specific funding was obtained for this study.

\section{Availability of data and materials}

All raw data is available from the corresponding author on request.

\section{Authors' contributions}

MY conceived and designed the study, recruited the participants, performed the literature review, collected and analyzed the data, and wrote the manuscript. $\mathrm{NN}, \mathrm{TT}$, and SN collected data and reviewed the manuscript. All authors read and approved the final manuscript.

\section{Ethics approval and consent to participate}

Ethical approval was obtained from the institutional review board of the host hospital. (Kyowakai Research Ethics Board \#16-02). Written informed consent to participate in the study was obtained from all the participants.

\section{Consent for publication}

Not applicable.

\section{Competing interests}

The authors declare that they have no competing interests.

\section{Publisher's Note}

Springer Nature remains neutral with regard to jurisdictional claims in published maps and institutional affiliations.

Received: 27 November 2018 Accepted: 7 March 2019

Published online: 01 April 2019

\section{References}

American Diabetes Association (2017) 2. Classification and diagnosis of diabetes. Diabetes Care 40:S11-S24

Boom DT, Sechterberger MK, Rijkenberg S, Kreder S, Bosman RJ, Wester JP, van Stijn I, DeVries JH, van der Voort PH (2014) Insulin treatment guided by subcutaneous continuous glucose monitoring compared to frequent pointof-care measurement in critically ill patients: a randomized controlled trial. Crit Care 18:453
Caplin NJ, O'Leary P, Bulsara M, Davis EA, Jones TW (2003) Subcutaneous glucose sensor values closely parallel blood glucose during insulin-induced hypoglycemia. Diabet Med 20:238-241

Danne T, Nimri R, Battelino T, Bergenstal RM, Close KL, DeVries JH, Garg S, Heinemann L, Hirsch I, Amiel SA, Beck R, Bosi E, Buckingham B, Cobelli C, Dassau E, Doyle FJ 3rd, Heller S, Hovorka R, Jia W, Jones T, Kordonouri O, Kovatchev B, Kowalski A, Laffel L, Maahs D, Murphy HR, Nørgaard K, Parkin CG, Renard E, Saboo B, Scharf M, Tamborlane WW, Weinzimer SA, Phillip M (2017) International consensus on use of continuous glucose monitoring. Diabetes Care 40:1631-1640

Hwang JS, Kim SJ, Bamne AB, Na YG, Kim TK (2015) Do glycemic markers predict occurrence of complications after total knee arthroplasty in patients with diabetes? Clin Orthop Relat Res 473(5):1726-1731

Jämsen E, Nevalainen P, Eskelinen A, Huotari K, Kalliovalkama J, Moilanen T (2012) Obesity, diabetes, and preoperative hyperglycemia as predictors of periprosthetic joint infection: a single-center analysis of 7181 primary hip and knee replacements for osteoarthritis. JBJS Am 94:e101

Keenan DB, Mastrototaro JJ, Voskanyan G, Steil GM (2009) Delays in minimally invasive continuous glucose monitoring devices: a review of current technology. J Diabetes Sci Technol 3:1207-1214

Kunutsor SK, Whitehouse MR, Blom AW, Beswick AD, INFORM Team (2016) Patient-related risk factors for Periprosthetic joint infection after Total joint arthroplasty: a systematic review and meta-analysis. PLoS One 11:e0150866

Madhu SV, Muduli SK, Avasthi R (2013) Abnormal glycemic profiles by CGMS in obese first-degree relatives of type 2 diabetes mellitus patients. Diabetes Technol Ther 15:461-465

Mraovic B, Suh D, Jacovides C, Parvizi J (2011) Perioperative hyperglycemia and postoperative infection after lower limb arthroplasty. J Diabetes Sci Technol 5:412-418

Nishimura R, Tsujino D, Taki K, Morimoto A, Tajima N, Nishimura R (2008) Does $\mathrm{HbA1c}$ represent a valid index for tight control of glucose in type 1 diabetes? Diabetes Res Clin Pract 82:e23-e24

O'Hara LM, Thom KA, Preas MA (2018) Update to the Centers for Disease Control and Prevention and the healthcare infection control practices advisory committee guideline for the prevention of surgical site infection (2017): a summary, review, and strategies for implementation. Am J Infect Control Mar 7

Rasbach LE, Atkins AE, Milaszewski KM, Keady J, Schmidt LM, Volkening LK, Laffel LM (2014) Treatment recommendations following 3-day masked continuous glucose monitoring (CGM) in youth with type 1 diabetes. J Diabetes Sci Technol 8:494-497

Rohlfing CL, Wiedmeyer HM, Little RR, England JD, Tennill A, Goldstein DE (2002) Defining the relationship between plasma glucose and $\mathrm{HbA}(1 \mathrm{c})$ : analysis of glucose profiles and $\mathrm{HbA}(1 \mathrm{c})$ in the diabetes control and complications trial. Diabetes Care 25:275-278

Sato H, Hosojima M, Ishikawa T, Aoki K, Okamoto T, Saito A, Tsuchida M (2017) Glucose variability based on continuous glucose monitoring assessment is associated with postoperative complications after cardiovascular surgery. Ann Thorac Cardiovasc Surg 23:239-247

Schierenbeck F, Franco-Cereceda A, Liska J (2017) Accuracy of 2 different continuous glucose monitoring Systems in Patients Undergoing Cardiac Surgery. J Diabetes Sci Technol 11:108-116

Shohat N, Tarabichi M, Tischler EH, Jabbour S, Parvizi J (2017) Serum Fructosamine: a simple and inexpensive test for assessing preoperative glycemic control. J Bone Joint Surg Am 99(22):1900-1907

Stryker LS, Abdel MP, Morrey ME, Morrow MM, Kor DJ, Morrey BF (2013) Elevated postoperative blood glucose and preoperative hemoglobin A1C are associated with increased wound complications following total joint arthroplasty. J Bone Joint Surg Am 95:808-814

Su G, Mi SH, Li Z, Tao H, Yang HX, Zheng H (2013) Prognostic value of early inhospital glycemic excursion in elderly patients with acute myocardial infarction. Cardiovasc Diabetol 12:33

Suwa T, Ohta A, Matsui T, Koganei R, Kato H, Kawata T, Sada Y, Ishii S, Kondo A, Murakami K, Katabami T, Tanaka Y (2010) Relationship between clinical markers of glycemia and glucose excursion evaluated by continuous glucose monitoring (CGM). Endocr J 57:135-140

Tarabichi M, Shohat N, Kheir MM, Adelani M, Brigati D, Kearns SM, Patel P, Clohisy JC, Higuera CA, Levine BR, Schwarzkopf R, Parvizi J, Jiranek WA (2017) Determining the threshold for $\mathrm{HbA1C}$ as a predictor for adverse outcomes after total joint arthroplasty: a multicenter, retrospective study. J Arthroplast 32:263-267

Zhou T, Dickson JL, Shaw GM, Chase JG (2017) Continuous glucose monitoring measures can be used for glycemic control in the ICU: an in-silico study. J Diabetes Sci Technol 12:7-19 